\title{
Vestibular function in severe bilateral vestibulopathy
}

\author{
G Wiest, J L Demer, J Tian, B T Crane, R W Baloh
}

\begin{abstract}
Objectives-To assess residual vestibular function in patients with severe bilateral vestibulopathy comparing low frequency sinusoidal rotation with the novel technique of random, high acceleration rotation of the whole body.

Methods-Eye movements were recorded by electro-oculography in darkness during passive, whole body sinusoidal yaw rotations at frequencies between 0.05 and $1.6 \mathrm{~Hz}$ in four patients who had absent caloric vestibular responses. These were compared with recordings using magnetic search coils during the first $100 \mathrm{~ms}$ after onset of whole body yaw rotation at peak accelerations of $2800^{\circ} / \mathrm{s}^{2}$. Off centre rotations added novel information about otolithic function.
\end{abstract}

Results-Sinusoidal yaw rotations at 0.05 $\mathrm{Hz}$, peak veocity $240 \%$ sielded minimal responses, with gain (eye velocity/head velocity) $<0.02$, but gain increased and phase decreased at frequencies between 0.2 and $1.6 \mathrm{~Hz}$ in a manner resembling the vestibulo-ocular reflex. By contrast, the patients had profoundly attenuated responses to both centred and eccentric high acceleration transients, representing virtually absent responses to this powerful vestibular stimulus.

Conclusion-The analysis of the early ocular response to random, high acceleration rotation of the whole body disclosed a profound deficit of semicircular canal and otolith function in patients for whom higher frequency sinusoidal testing was only modestly abnormal. This suggests that the high frequency responses during sinusoidal rotation were of extravestibular origin. Contributions from the somatosensory or central predictor mechanisms, might account for the generation of these responses. Random, transient rotation is better suited than steady state rotation for quantifying vestibular function in vestibulopathic patients.

(f Neurol Neurosurg Psychiatry 2001;71:53-57)

Keywords: bilateral vestibulopathy; vestibulo-ocular reflex; high acceleration rotation

Patients with absent caloric vestibular responses often have robust compensatory eye movements in darkness at higher frequencies of sinusoidal rotation. ${ }^{12}$ It is unclear whether these eye movements arise from the vestibular labyrinths, or some other sensory system. The predictability of sinusoidal head motion might allow subjects to generate responses synchronised to non-vestibular sensory cues. Experimental studies in animals showed that bilaterally labyrinthectomised monkeys develop preprogrammed compensatory eye movements in anticipation of a head movement. ${ }^{3}$

A powerful method to quantify reliably the vestibulo-ocular reflex (VOR) without contamination by non-vestibular mechanisms involves testing during random transient rotations at high acceleration. Although the direct response of labyrinthine afferents is vital to generation of the short latency VOR, considerable central processing affects the late and steady state VOR. As central processing takes time, the basic labyrinthine response can be isolated by examining the earliest response to transient head rotation. When visually salient targets are presented within a few 10 s of milliseconds before (or even during) transient rotation, VOR responses during the first $100 \mathrm{~ms}$ are highly repeatable within individual subjects and yet attributable only to labyrinthine input.

Previous rotational studies in normal human subjects used whole body accelerations only in the range of $300 \% \mathrm{~s}^{2}$ to $1000 \% \mathrm{~s}^{2}{ }^{45}$ In the present experiments, patients were exposed to whole body peak accelerations of $2800^{\circ} / \mathrm{s}^{2}$, as previous studies showed that the initial VOR depends qualitatively and quantitatively on the magnitude of head acceleration. ${ }^{6}$ To our knowledge, this technique has not yet been used to examine residual vestibular function in patients with bilateral vestibulopathy. We compared ocular responses to standard sinusoidal stimulation over a wide frequency range with responses to random, high acceleration transients of the whole body in four patients who lacked caloric responses. We investigated whether the dynamics of the sinusoidal compensatory eye movements were similar to those of the VOR, and whether they correlated with the magnitude of the responses evoked by unpredictable, high acceleration transients.

\section{Methods}

PATIENTS

Four patients with bilateral vestibulopathy and absent caloric responses were selected. All patients had dysequilibrium, worse in the dark, and prominent oscillopsia. As none had associated hearing loss, history of ophthalmological disorders, exposure to known ototoxins, or a positive family history, the patients were diagnosed as having idiopathic bilateral vestibulopathy. ${ }^{7}$ None had spontaneous or gaze evoked nystagmus while fixating. Their average age was 52 (SD 17) years, range $39-78$ years. The interval between onset of symptoms and time of testing varied from 2 months to 2 years. 
Table 1 Gain values for high acceleration transients and sinusoidal rotation in patients and normal controls

\begin{tabular}{llll}
\hline & \multicolumn{2}{l}{ Mean transient gain } & \\
\cline { 2 - 3 } & $\begin{array}{l}\text { Centred rotations 25-100 } \\
\text { ms }\end{array}$ & $\begin{array}{l}\text { Eccentric rotations } \\
25-100 \mathrm{~ms}\end{array}$ & $\begin{array}{l}\text { Sinusoidal gain } 0.8 \\
\mathrm{~Hz}\end{array}$ \\
\hline Patient 1 & $0.05(0.006)$ & $0.12(0.007)$ & 0.58 \\
Patient 2 & $0.27(0.004)$ & $0.29(0.005)$ & 0.72 \\
Patient 3 & $0.04(0.007)$ & $0.08(0.006)$ & 0.30 \\
Patient 4 & $0.03(0.005)$ & $0.03(0.005)$ & 0.42 \\
Normal subjects & $0.88(0.002) \ddagger$ & $0.95(0.002) \ddagger$ & $0.71(0.18) \dagger$ \\
\hline
\end{tabular}

Transient gain values are mean $(\mathrm{CW}+\mathrm{CCW}) / 2 \mathrm{SEM})$.

*Normal controls $(\mathrm{n}=11)$.

†Normal mean (SD) (Baloh et al, Ann Neurol 1988;23:32-7).

$\ddagger$ Normal mean $(\mathrm{SEM})^{6}$

TEST PROCEDURES

Patients were rotated sinusoidally in the horizontal plane in darkness with their eyes open, using a servomotor driven rotational chair. They performed mental arithmetic throughout the testing to maintain a constant state of alertness. All patients were rotated for $4-8$ cycles at $0.05 \mathrm{~Hz}$ (peak velocity 60,120 , and $240 \% \mathrm{~s}), 0.2 \mathrm{~Hz}(60 \%), 0.4 \mathrm{~Hz}(30 \%), 0.8$ $\mathrm{Hz}(30 \%)$, and $1.2 \mathrm{~Hz}(30 \%)$; two patients were additionally rotated at $1.6 \mathrm{~Hz}(30 \%)$.

During sinusoidal rotation, eye movements were recorded with electro-oculography digitally sampled at $200 \mathrm{~Hz}$. Calibration by fixation of targets at known eccentricities was performed before rotation at each test frequency. Recordings were simultaneously displayed on polygraph paper and analyzed on line by a digital computer.

High acceleration, transient, whole body yaw rotations were administered by a $500 \mathrm{~N}-\mathrm{m}$, high resolution stepper motor (Compumotor, Rohnert Park, CA, USA) with a dedicated driver and position feedback digital controller. A detailed description of the equipment has been published. ${ }^{6}$

Briefly, subjects sat in a wooden chair that was fitted with dense foam cushions, and their bodies were secured by lap and chest belts while their knees and feet were firmly held by padded braces. Subjects' heads were tightly secured within a non-metallic, conforming head holder. Directionally and temporally unpredictable transient yaw rotations were delivered about an axis centred between the otoliths to examine pure canal responses, and off centre $20 \mathrm{~cm}$ posterior to the eyes. Eccentric rotation provides simultaneous angular and linear motion and thus enables assessment of combined otolith and canal responses. Ten rotations were performed in random sequence in each direction. Subjects were instructed to fixate a target located 500 $\mathrm{cm}$ in front of them, consisting of a $14 \mathrm{~cm}$ black cross on a $102 \times 81 \mathrm{~cm}$ white background. Fifty to $70 \mathrm{~ms}$ before onset of each rotation, room lights were extinguished and subjects were instructed to maintain gaze on the remembered target. Peak acceleration was $2800 \% \mathrm{~s}^{2}$ to a maximum velocity of $190 \%$, which rotated the head $40^{\circ}$ in $250 \mathrm{~ms}$. Eccentric rotation yielded peak otolith tangential acceleration of $0.6 \mathrm{~g}$. Eye and head positions were measured with magnetic search coils (Skalar Medical, Delft, The Netherlands), placed on the right eye under topical anaesthesia, and on a bite bar moulded to the upper teeth, respectively. Search coil data (horizontal and vertical gaze and head position) were low pass filtered over a bandwidth of $300 \mathrm{~Hz}$ before simultaneous digital sampling at $1.2 \mathrm{kHz}, 16$ bit resolution.

DATA ANALYSIS

A detailed description of the on line digital computer analysis technique for the sinusoidal rotational responses has been published elsewhere. ${ }^{1}$ Eye position was differentiated to yield instantaneous eye velocity. Fast components were identified by their characteristic velocity profile and removed from the data. The gaps in the remaining slow phase eye velocity record were filled by interpolation with a quadratic regression line. A fast Fourier transform was executed giving the magnitude and phase of the fundamental and first five harmonics. As the responses were roughly symmetric, the gain and phase were calculated by comparing the fundamental with the stimulus velocity trace. Only responses with coherence $>0.95$ were accepted for evaluation. Time constants were calculated from sinusoidal rotational responses. Calculation of the time constant from constant velocity rotational testing was not possible due to absence of postrotatory nystagmus in all patients, consistent with the ultrashort time constants found in the sinusoidal responses.

A detailed description of the methods for analyzing high acceleration responses appears elsewhere. ${ }^{6}$ Data were analyzed using the MacEyeball custom software package running under LabView (National Instruments, Austin, TX,USA). For each subject, rotations were grouped based on direction and rotation axis. Events in which eye position varied by $>0.2^{\circ}$ in the $80 \mathrm{~ms}$ preceding head rotation were discarded as failures of fixation. After digital differentiation, eye and head velocity data were filtered using a third order low pass Butterworth filter $(0-50 \mathrm{~Hz})$. Gain was determined by dividing instantaneous eye velocity by head velocity. Right eye position was defined as zero before the chair began moving because the target was directly in front of the right eye. Normative data have been reported for both types of rotational stimuli. ${ }^{6}$

\section{Results}

RESPONSES TO SINUSOIDAL ROTATION

Sinusoidal rotation of vestibulopathic subjects at $0.05 \mathrm{~Hz}$ yielded minimal responses (gain $<0.02$ ) even at peak velocities of $240 \%$ s. The VOR gain increased with increasing frequency in all patients, ranging from 0.030.15 at $0.2 \mathrm{~Hz}$ and from $0.43-0.70$ at $1.6 \mathrm{~Hz}$. By contrast, phase lead decreased with higher frequencies. At $0.2 \mathrm{~Hz}$ phase lead ranged from $41^{\circ}-59^{\circ}$ in all patients, decreasing to a range of $21^{\circ}-34^{\circ}$ at $0.8 \mathrm{~Hz}$. Figure 1 shows electrooculographic recordings of all patients at 0.2 and $0.8 \mathrm{~Hz}$. Mean gain at $0.8 \mathrm{~Hz}$ was 0.50 (SD 0.18 ) (table 1) and mean phase lead was 30.9 (SD 10) ${ }^{\circ}$, yielding a mean time constant of $0.36($ SD 0.13$) \mathrm{s}$ (range $0.21-0.51 \mathrm{~s}$ ) at $0.8 \mathrm{~Hz}$. Gain and phase values at higher frequencies 
(a)
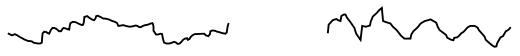

(b)

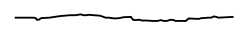

(c)

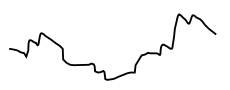

(d)
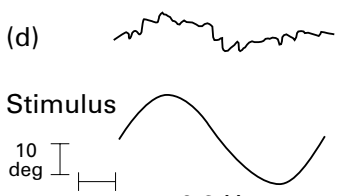

$1 \mathrm{sec}$

$0.2 \mathrm{~Hz}$
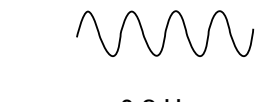

Figure 1 Electro-oculographic recordings of eye position during sinusoidal rotation of patient 1 (a), 2 (b), 3 (c), and 4 (d) at 0.2 and $0.8 \mathrm{~Hz}$. At $0.8 \mathrm{~Hz}$ mostly smooth compensatory eye movements occurred. Responses at 0.2 $\mathrm{Hz}$ clearly show quick and slow phases, consistent with reflexive eye movements.
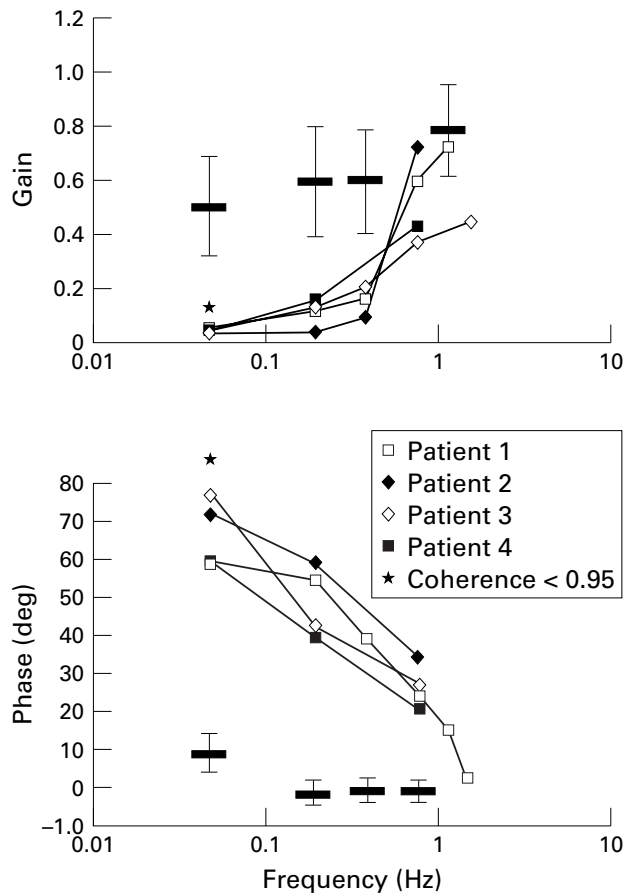

Figure 2 Bode plots of gain and phase for sinusoidal VOR at $0.05,0.2,0.4,0.8,1.2$ and $1.6 \mathrm{~Hz}$ in all patients. The bold horizontal lines and the vertical bars indicate the normal mean (SD). Note the increase in gain and the decrease of phase in the patients' responses at higher frequencies, corresponding to a first order linear model of the VOR. ${ }^{\star}$ Coherence $<0.95$.

were consistent with a 1 st order high pass linear system. Figure 2 plots the gain and phase of responses to sinusoidal stimulation compared with normal subjects. Phase lead was abnormally large at low frequencies, but at the highest frequencies phase lead approached the normal value of zero. Slow compensatory and fast anticompensatory eye movements were present at $0.2 \mathrm{~Hz}$ (fig 1 ).

\section{RESPONSES TO TRANSIENT HIGH ACCELERATION} ROTATION

Figure 3 shows responses to transient rotations centred between the otoliths, so that net otolith stimulation was minimal. The responses of each patient and a normal subject (normal data taken from Crane and Demer ${ }^{6}$ ) are shown for
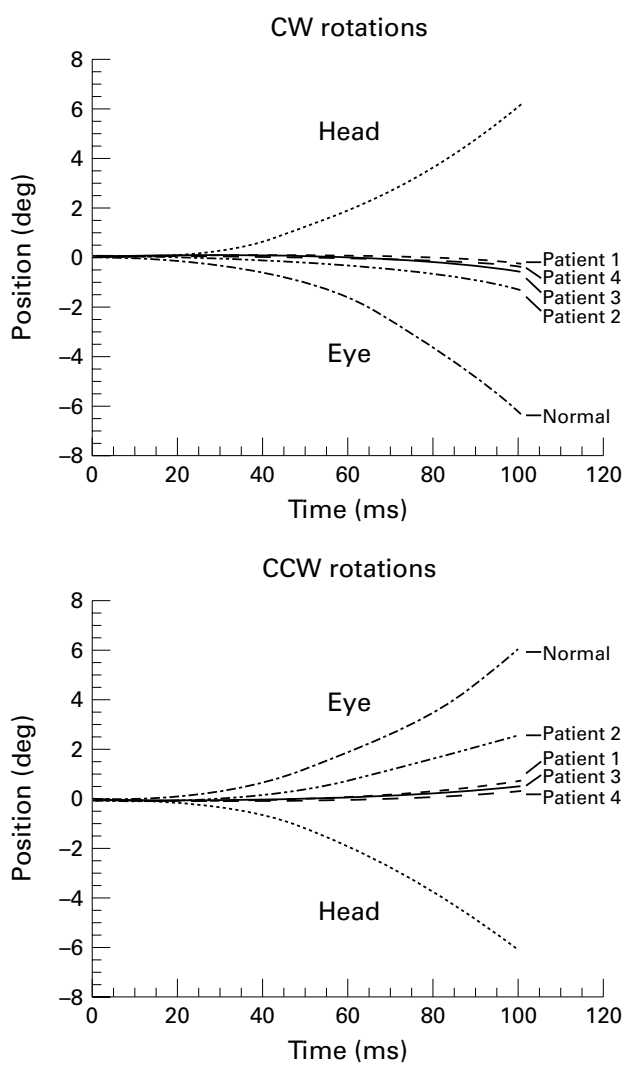

Figure 3 Magnetic search coil recordings of eye and head position during the initial $100 \mathrm{~ms}$ of transient rotation to peak acceleration $2800^{\circ} / \mathrm{s}^{2}$. Each plot represents the average (SEM) of 10 rotations centred at the interaural point in a normal subject (normal) and in each patient (patients 1-4) for clockwise $(C W)$ and counter clockwise $(C C W)$ directions. Patient 2 showed the highest response bilaterally, suggesting residual vestibular function. Responses were minimal for patients 1, 3, and 4. Error limits were typically indistinguishable from data traces.

clockwise and counter clockwise rotations. Each trace represents averages of 8-10 rotations under identical conditions. By comparison with normal controls, responses of vestibulopathic subjects both to centred and eccentric rotations were almost abolished (table 1 ). The VOR gain for rotations centred between the otoliths averaged over the interval $25 \mathrm{~ms}-100$ $\mathrm{ms}$ after rotation onset ranged from 0.02 to 0.35 for both directions. A shift in axis eccentricity to $20 \mathrm{~cm}$ posterior to the eyes creates a strong otolith stimulus synergistic with the angular VOR that normally increases gain markedly; yet in the patients there was only a minimal increase in gain (table 1). Gain during eccentric rotations $25 \mathrm{~ms}-00 \mathrm{~ms}$ after rotation onset ranged from 0.01 to 0.49 for both directions, with slow phase eye velocities ranging from $2.2 \%$ to $46 \%$ s. Mean gain values of each patient are compared with normal controls in the table 1 . Patient 2 had some residual VOR (fig 3) to transient rotation. Surprisingly, this patient had the lowest sinusoidal gain at $0.2 \mathrm{~Hz}$ and the highest gain at $0.8 \mathrm{~Hz}$ (fig 2).

\section{Discussion}

The current study examined reflexive eye movements in response to random, transient high accelerations of the whole body about centred and eccentric axes, demonstrating a 
profound deficit of the VOR to both semicircular canal and otolith stimulation in patients with bilateral loss of caloric responsiveness. Conventional "impulse" rotational tests have been performed using whole body rotations to constant velocity, with eye movement recording by electro-oculography. ${ }^{9}$ These tests measure the slow component velocity of nystagmus after step changes in angular velocity and provide assessment of gain and the time constant of the VOR. As these tests use 20-fold lower accelerations of about $140 \% \mathrm{~s}^{2}$ and as slow component velocities of the VOR are measured in a time window of up to 30 seconds, ${ }^{10}$ the responses are not comparable with the high acceleration transients of $2800 \% \mathrm{~s}^{2}$ employed in the present study. The response in the first 100 $\mathrm{ms}$ after onset of high acceleration rotation is likely to be a pure vestibular response as there is insufficient time for other systems (for example, vision, somatosensory) to contribute, making this stimulus an ideal tool for the assessment of vestibular function.

Previous studies have achieved high head accelerations using manually delivered thrusts of the head on the trunk to evaluate deficits of unilateral vestibular lesions. ${ }^{11}{ }^{12}$ Such rotations are inherently more variable across time, experimenters, and subjects. Tabak et al induced passive step displacements of the head by a reactive torque helmet in patients with absent caloric responses. ${ }^{13}$ These authors found that the magnitude of the gain reduction correlated well with the degree of disability. However, transient head velocities in these patients only reached $55 \%$, by comparison with $190 \%$ in our experiments, and the relatively high average gain of about 0.48 for transient head rotation was attributed by the authors to inhomogeneity and partial lesions in their patient population.

High acceleration transient rotations of the whole body at $3000 \% \mathrm{~s}^{2}$ have been recently used to examine the response dynamics of the VOR both in normal monkeys and in monkeys after unilateral plugging of the semicircular canals. ${ }^{14}{ }^{15}$ Residual vestibular function in vestibulopathic patients has not to our knowledge been evaluated with this method. By contrast with passive head on trunk rotation, transient whole body acceleration is not confounded by additional neck receptor stimulation and provides a direct comparison with the sinusoidal responses in standard rotational testing. The VOR gain in our patients $25-100 \mathrm{~ms}$ after onset of the high acceleration transients ranged from 0.05 to 0.27 , being minimally affected by shifting the rotational axis off centre. This examination confirmed essentially absent semicircular canal and otolithic function in patients 1,3 , and 4 , calling into question the origin of the sinusoidal rotational responses.

Sinusoidal rotational testing in our patients with bilateral vestibulopathy showed a markedly decreased sensitivity to low frequency stimulation, consistent with previous studies. ${ }^{12}$ The responses at higher frequencies showed VOR-like dynamics of increasing gain and decreasing phase lead with increasing frequency, but had remarkably short time con- stants. Shortening of the VOR time constant has previously been reported only in patients with CNS lesions. ${ }^{816}$ If the sinusoidal rotational responses (which probe the VOR in the frequency domain) are of vestibular origin, they should correlate with the responses to the high acceleration impulses, which probe the VOR in the time domain..$^{11} 18$

Compensatory slow eye movements have also been reported in a patient with bilateral vestibular nerve section. ${ }^{19}$ Passive, unpredictable transient head movements in this patient evoked smooth compensatory eye movements of low gain (about 0.3 ), which were thought to be generated by cervical sources. During whole body rotation, this patient was instructed to maintain gaze on the location of an earth fixed light after it had been extinguished. The authors concluded that the compensatory eye movements in this test were produced by pursuit of the imaginary fixation light. Maintaining gaze on an imagined earth fixed target during sinusoidal rotation in darkness requires a central percept of head velocity to generate appropriate compensatory eye movements, and some sensory information about self motion. Although none of our patients was instructed to fixate or imagine the location of an earth fixed target during sinusoidal rotation, it is conceivable that they might have used extravestibular cues and predictor mechanisms.

Experimental studies confirmed that normal subjects could generate smooth pursuit of predictable, imaginary, or invisible stimuli in darkness. $^{2021}$ Smooth eye movements can occur in anticipation of a target motion even when the stimulus does not appear and a period as long as 4 seconds has elapsed from the previous visual stimulus. ${ }^{22}$ Smooth pursuit and fixation of an imagined earth fixed target during sinusoidal rotation are achieved by the same neural mechanisms. ${ }^{23}$

Even if we assume the efficiency of predictor mechanisms in generating compensatory eye movements in our patients, there must still be another mechanism indicating the temporal characteristics of the stimulus. In the absence of vestibular cues, a likely source for this information might be the somatosensory system. Recent experiments provide evidence that the timing and the velocity storage of anticipatory smooth pursuit eye movements can be independently controlled through different sensory channels. $^{24}$ Although anatomical and physiological findings strongly suggest a somatosensory input to the vestibular nuclei, ${ }^{25-28}$ the functional relevance of these afferents has not yet been established. A recent study confirmed modulation of vestibular nucleus activity during dynamic tilt in decerebrate cats lacking vestibular and neck somatosensory inputs. ${ }^{29}$ The authors suggested that modulation of vestibular nucleus neuronal activity in these animals was due to activation of receptors in the viscera, trunk, or limbs.

So far, there are only a few studies dealing with the functional role of the somatosensory system in patients with bilateral vestibulopathy. Assessing purely somatosensory responses in normal subjects is impossible with conven 
cctional rotational stimuli. Apparent stepping around on a moving platform (that is, if a stationary subject steps on a rotating platform) stimulates exclusively the somatosensory system without activating the labyrinths, producing circular vection and somatosensory nystagmus. In this paradigm, patients with defective labyrinths exhibit a stronger somatosensory induced nystagmus than normal subjects. ${ }^{30}$ The existence of so-called arthrokinetic nystagmus is further evidence for a functionally significant somatosensory-vestibular convergence. ${ }^{31}$ Our findings suggest that high acceleration rotations of the whole body may be superior to sinusoidal rotational testing in the assessment of residual vestibular function. The severe degradation of the VOR to transient high whole body acceleration suggests that the responses to high frequency sinusoidal rotations in our patients were of extravestibular origin in at least three of four patients. It is likely that the responses to sinusoidal rotation were due to somatosensory or other extravestibular sensory origins, probably enhanced by central predictor mechanisms.

We thank N DeSalles, L Fleischman, and F Henriquez for technical assistance. The work was supported by NIH grant P01 DC02952 and the Austrian Science Fund. JLD was the recipient of a Lew R Wasserman Merit Award from Research to Prevent Blindness, and is the Laraine and David Gerber Professor of Ophthalmology.

1 Baloh RW, Honrubia V, Yee RD, et al. Changes in the human vestibulo-ocular reflex after loss of peripheral sensitivity. Ann Neurol 1984;16:222-8.

2 Honrubia V, Marco J, Andrews J, et al. Vestibulo-ocular reflex in peripheral labyrinthine lesions: bilateral dysfunction. Am 7 Otolaryngol 1985;6:342-52.

3 Dichgans J, Bizzi E, Morasso P, et al. Mechanisms underlying recovery of eye-head coordination after bilateral labyrinthectomy in monkeys. Exp Brain Res 1973;18:54862 .

4 Anastasopoulos D, Gianna CC, Bronstein AM, et al. Interaction of linear and angular vestibulo-ocular reflexes of human subjects in response to transient motion. Exp of human subjects in respons

5 Crane BT, Viirre ES, Demer JL. The human horizontal vestbulo-ocular reflex during combined linear and angular vestbulo-ocular reflex during combined linear

6 Crane BT, Demer JL. Human horizontal vestibulo-ocular reflex initiation: effects of acceleration, target distance, and unilateral deafferentation. $\mathcal{F}$ Neurophysiol 1998;80:115166.

7 Baloh RW, Jacobson K, Honrubia V. Idiopathic bilateral vestibulopathy. Neurology 1989;39:272-5.

8 Baloh RW, Beykirch K, Tauchi P, et al. Ultralow vestibuloocular reflex time constants. Ann Neurol 1988;23:32-7.

9 Baloh RW, Honrubia V. Clinical neurophysiology of the vestibular system. 2nd ed. Philadelphia: FA Davis, 1990.
10 Baloh RW, Sakala SM, Yee RD, et al. Quantitative vestibular testing. Otolaryngol Head Neck Surg 1984;92:145-50.

11 Halmagyi GM, Curthoys IS, Cremer PD, et al. The human horizontal vestibulo-ocular reflex in response to high acceleration stimulation before and after unilateral vestibular neurectomy. Exp Brain Res 1990;81:479-90.

12 Halmagyi GM, Curthoys IS, Todd MJ, et al. Unilateral vestibular neurectomy in man causes a severe permanent horizontal vestibulo-ocular reflex deficit in response to high-acceleration ampullofugal stimulation. Acta Otolaryngol Suppl 1991;481:411-14.

13 Tabak S, Collewijn H, Boumans LJJM, et al. Gain and delay of human vestibulo-ocular reflexes to oscillation and steps f the head by a reactive torque helmet. II Vestibula deficient subjects. Acta Otolaryngol 1997;117:796-809.

14 Minor LB, Lasker DM, Backous DD, et al. Horizontal vestibuloocular reflex evoked by high acceleration rotations in the squirrel monkey. I. Normal responses. F Neurophysiol 1999;82:1254-70.

15 Lasker DM, Backous DD, Lysakowski A, et al. Horizontal vestibuloocular reflex evoked by high acceleration rotations in the squirrel monkey. II. Responses after canal plugging. 7 Neurophysiol 1999;82:1271-85.

16 Demer JL, Zee DS. Vestibulo-ocular and optokinetic deficits in albinos with congenital nystagmus. Invest Ophthalmol Vis Sci 1984;25:739-45.

17 Foster CA, Demer JL, Morrow MJ, et al. Deficits of gaze stability in multiple axes following unilateral vestibular lesions. Exp Brain Res 1997;116:501-9.

18 Gilchrist DPD, Curthoys IS, Cartwright AD, et al. High acceleration impulsive rotations reveal severe long term deficits of the horizontal vestibulo-ocular reflex in the guinea pig. Exp Brain Res 1998;123:242-54.

19 Halmagyi GM, Curthoys IS. Human compensatory slow eye movements in the absence of vestibular function. In: Graham MD, Kemink JL, eds. The vestibular system: neurophysiologic and clinical research. New York: Raven Press, 1987.

20 Gauthier GM, Hofferer JM. Eye tracking of self moved targets in the absence of vision. Exp Brain Res 1976;26:12139 .

21 Whittaker SG, Eaholtz G. Learning patterns of eye motion for foveal pursuit. Invest Ophthalmol Visual Sci 1982;23: 393-7.

22 Barnes GR, Asselman PT. The mechanism of prediction in human smooth pursuit eye movements. F Physiol 1991;439: 439-61.

23 Barnes GR, Eason RD. Effects of visual and non-visual mechanisms on the vestibulo-ocular reflex during pseudomechanisms on the vestibulo-ocular reflex during pseudo-
random head movements in man. $\mathcal{F}$ Physiol 1988;395:383400.

24 Barnes GR, Donelan SF. The remembered pursuit task: evidence for segregation of timing and velocity storage in predictive oculomotor control. Exp Brain Res 1999;129:57-67.

25 Wilson VJ, Kato M, Thomas RS, et al. Excitation of lateral vestibular neurons by peripheral afferent fibers. I Neurophysiol 1966;29:508-29.

26 Wilson VJ, Wylie RM, Marco LA. Synaptic inputs to cells in the medial vestibular nucleus. F Neurophysiol 1968;31:17685.

27 Pompeiano O. Spinovestibular relations: anatomical and physiological aspects. Prog Brain Res 1972;37:263-96.

28 Rubin AM, Liedgren SR, Odkvist LM, et al. Limb input to the cat vestibular nuclei. Acta Otolaryngol 1979;87:113-22.

29 Yates BJ, Jian BJ, Cotter LA, et al. Responses of vestibular nucleus neurons to tilt following chronic bilateral removal of vestibular inputs. Exp Brain Res 2000;130:151-8.

30 Bles W, Vianney de Jong JMB, de Wit G. Somatosensory compensation for loss of labyrinthine function. Acta Otolaryngol 1984;97:213-21.

31 Brandt Th, Buechele W, Arnold F. Arthrokinetic nystagmus and ego-motion sensation. Exp Brain Res 1977;30:331-8. 